\title{
On Bit-loading for Discrete Multi-tone Transmission over Short Range POF Systems
}

\author{
Linning Peng, Maryline Hélard, and Sylvain Haese
}

\begin{abstract}
A novel bit-loading approach is proposed for the discrete multi-tone (DMT) transmission over short range polymer optical fiber (POF) systems. First of all, from the extract signal-to-noise ratio (SNR) table of quadrature amplitude modulation (QAM) for different desired bit error rates (BER), a new linear approximation (LA) expression is introduced to implement bit-loading for DMT systems. Then, based on water-filling concept, the performance bounds and optimal power allocations for the classical and the proposed bit-loading algorithms in Gaussian low-pass channel models are derived. Consequently, introducing the measured channel parameters of step-index (SI)-POF channels with different transmission distances, the theoretical performance bounds are computed and the practical transmission rates are simulated. Simulation results show that the proposed LA expression based bit-loading achieves higher transmission rate than classical modulation gap based bit-loading. Both algorithms use sub-optimal Chow algorithm with constant power allocation and iterative process. Finally, real DMT transmissions over SI-POFs are implemented in order to verify the proposed method. The LA expression based bit-loading outperforms the modulation gap based bit-loading in DMT transmission systems over different transmission distances. Moreover, experimental results show that the longer fiber length, the higher performance gain with LA expression based bit-loading. In the comparisons, for a $50 \mathrm{~m}$ SI-POF transmission, the transmission rate in DMT system with LA expression based bit-loading is improved by $5 \%$ with the same experimental setups for a given BER at $1 \times 10^{-3}$ and by $10 \%$ for a $100 \mathrm{~m}$ length.
\end{abstract}

Index Terms-POF, RCLED, DMT, OFDM, bit-loading, water-filling, power allocation, channel capacity, channel model

\section{INTRODUCTION}

$\mathrm{T}$ HE $\varnothing 1 \mathrm{~mm}$ large core diameter step-index (SI-) polymer optical fiber (POF) has the advantages of low cost and easy installation, making it interesting for indoor networking [1]. However, compared to the traditional silica optical fiber, the transmission bandwidth in the POF is limited [2]. In recent years, the discrete multi-tone (DMT) transmissions over POFs have attracted more and more research interests [3][4][5]. Being similar to the well-know orthogonal frequency division multiplexing (OFDM), DMT modulation is a baseband multi-carrier modulation (MCM) technique, which has been

The authors are with Institute of IETR (Electronic and Telecommunications in Rennes), INSA (National Institute of Applied Sciences) of Rennes, Rennes 35700, France (E-mails: \{linning.peng, maryline.helard, sylvain.haese\}@insa-rennes.fr). already used in asymmetric digital subscriber line (ADSL) for decades [6]. The work carried out in the framework on the European project plastic optical fiber for pervasive low-cost ultra-high capacity systems (POF-PLUS) [7] allowed for a 1 Gbps transmission over $50 \mathrm{~m}$ SI-POF by using DMT modulation [8][9].

The intensity modulation and direct detection (IM/DD) was largely adopted in POF transmission systems under low-cost consideration [3]. Several published works demonstrated that the SI-POF transmission channel can be modeled as a Gaussian low-pass channel [2][10]. The theoretical channel capacity of the SI-POF systems employing DMT were investigated by [3][11][10]. In a practical DMT transmission, the bit-loading algorithms were largely adopted in order to approach the channel capacity [8][9]. In most of the published works, the Levin-Campello (LC) algorithm [12]13], and the sub-optimal Chow algorithm [14] were used [5][8][9][15].

In Chow algorithms, a concept of modulation gap $\Gamma$ was introduced to build the link between the theoretical channel capacity and practical transmission rate. The authors of [10] presented the $\Gamma$ for different desired symbol error rates (SER). In LC algorithm, an extract SNR table based bit-loading algorithm was proposed in order to maximize the transmission rate [13]. Furthermore, Czylwik proposed a linear approximation of the required signal-to-noise ratio (SNR) for the required SER in bit-loading of OFDM systems [16], which can give a better bit-loading approximation than the modulation gap in low SNR conditions. The performance of modulation gap based bit-loading with LC algorithm and Chow algorithms were investigated by [10]. Both the LC and Chow algorithms can provide similar transmission data rates in different SI-POF channel models [10].

In DMT transmissions, the conventional modulation gap based bit-loading was used to reach a targeted SER when the SNR at each subcarrier is measured by probing DMT symbols. However, a targeted bit error rate (BER) is normally required to ensure the quality of the transmission by means of a channel coding. An exact calculation of the BER would take into account the constellation and the applied mapping of the modulation. Thus the difference between the use of the classical SER evaluation or of a better BER evaluation will lead to some deviations in terms of transmission rate and used bandwidth after bit-loading.

In this paper, we propose a more efficient BER-based approach for bit-loading in DMT transmissions. We establish exact BER performance for different quadrature amplitude 
modulation (QAM). The main contributions of this paper are firstly the use of a linear approximation (LA) of the BER performance for different QAM in the bit-loading algorithm and secondly the derivation of the performance bounds and optimal power allocations. Both the classical and the proposed bit-loading algorithms using a theoretical channel model are studied and compared. Furthermore, we verify the proposed system by real experiments for different transmission distances.

In Section II, we present the principle of LA expression bit-loading for DMT transmission. The parameters of the LA expressions for different QAM and desired BER are presented. In Section III, the Gaussian low-pass channel model is introduced to approach the practical SI-POF channel. The performance bounds and optimal power allocation of the proposed LA expression and the classical modulation gap based bit-loadings are theoretically investigated in terms of water-filling optimization. This theoretical approach is therefore used to evaluate the performance of practical bit-loading. Then Section IV introduces the practical iterative bit-loading process with LA expression and modulation gap. In Section V, we build an experimental SI-POF system with a low-cost resonant cavity light emitting diode (RCLED) and obtain the channel models for different POF lengths. In Section VI, we present the simulation results of theoretical performance bounds and practical available transmission rates for different targeted BER and POF length. In Section VII, in order to verify the performance, real SI-POF transmission system with different POF lengths are tested by DMT modulation employing the proposed LA expression and the classical modulation gap based bit-loadings. Finally, the conclusion and prospect are given in Section VIII.

\section{SNR TABLE BASED LINEAR APPROXIMATION}

\section{A. Bit-loading from Modulation Gap}

In most of the DMT transmissions over POF, a different number of information bits is allocated to each subcarrier [5][8][9]. In general, the allocated bits $b$ require an $2^{b}$-order QAM modulation. With the Gray mapping, the square constellation maps are usually used for even order QAM, such as 4-QAM, 16-QAM, 64-QAM, 256-QAM and 1024-QAM and the rectangle constellation maps are usually used for 2-PSK and 8-QAM. Furthermore, the cross constellation maps are usually used for odd order QAM with higher modulations [17], such as 32-QAM, 128-QAM and 512-QAM. An example of Gray mapping for 4-QAM, 8-QAM, 16-QAM and 32-QAM are presented in Fig. 1.

Shannon's formula is well-known for the capacity of a band-limited Gaussian channel [18]. In practical system, the modulation gap $\Gamma$ is the difference between the channel capacity and the real achievable transmission rate depending on error probability requirements [10]. In the bit-loading algorithms with the modulation gap $\Gamma$, the allocated bits $b$ for QAM modulation can be computed from the SNR:

$$
b=\log _{2}\left(1+\frac{10^{(\operatorname{SNR}(d B) / 10)}}{\Gamma}\right) .
$$

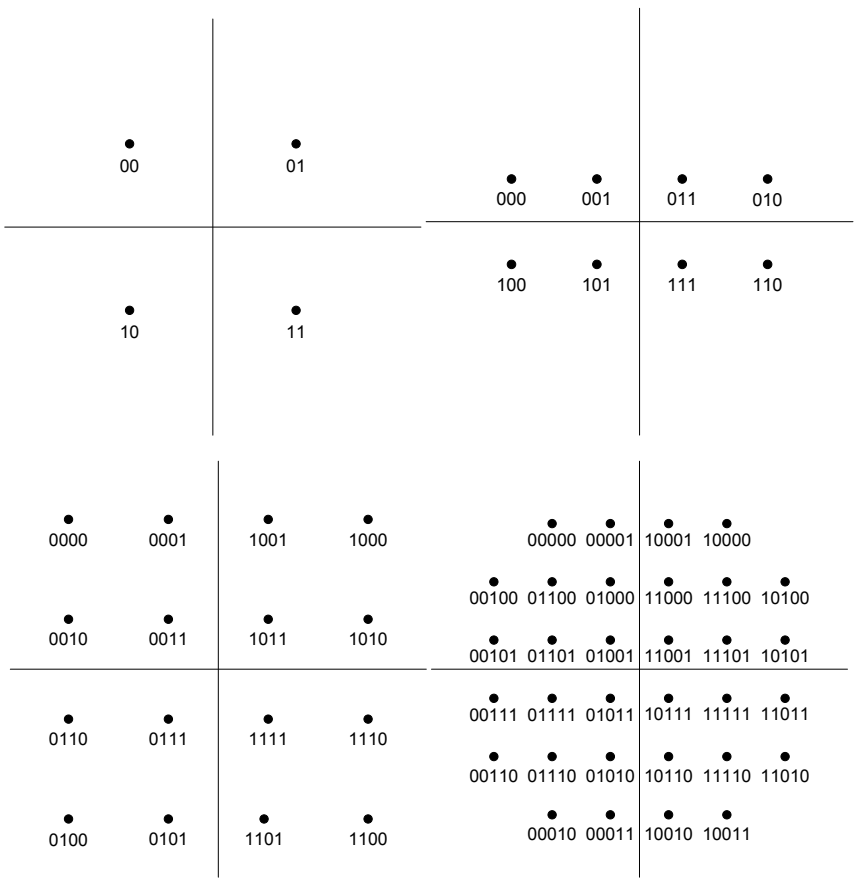

Fig. 1. An example of Gray mapping for square 4-QAM, rectangle 8-QAM, square 16-QAM and cross 32-QAM

In optical DMT systems, the bit-loading is performed for each subcarrier with the information from DMT probing symbols. After channel estimation with the DMT probing symbols, subcarrier SNR can be obtained. Therefore the SNR $(d B)$ in Eq. (1) is defined as the measured electrical SNR with the bandwidth of subcarrier frequency spacing. The $\operatorname{SNR}(d B)$ is in decibel $(\mathrm{dB})$ scale and the value of the $\mathrm{dB}$ scale is used in this paper. The modulation gap $\Gamma$ for QAM can be theoretically calculated from the determinate SER with the following formula [19]:

$$
\Gamma=\frac{1}{3}\left[\mathrm{Q}^{-1}\left(\frac{\mathrm{SER}}{4}\right)\right]^{2},
$$

the $Q$ function is defined as:

$$
\mathrm{Q}(x)=\int_{x}^{\infty} \frac{e^{-u^{2} / 2}}{\sqrt{2 \pi}} d u .
$$

The Eq. (1) and (2) are accurate for QAM with square and rectangular constellations. Although it is not always exact, it gives a good approximation to the bit loading algorithms [10][14][19].

However, in most of the optical DMT transmission systems employing bit-loading, the modulation orders show dramatic variations, such as from 2-PSK to 1024-QAM [8][9][20][21]. It is worth noting that when Gray mapping constellation is selected, the calculated SER is approximately $b$ times larger than the desired BER for high SNR. Therefore the SER oriented calculation of $\Gamma$ will cause offset when modulation orders show dramatic variations.

For instance, for a 16-QAM ( $b$ is 4$)$, with the desired BER at $1 \times 10^{-4}$, the SER for the modulation gap $\Gamma$ calculation is equivalent to $4 \times 10^{-4}$. The calculated modulation gap $\Gamma$ of the 16-QAM with different desired BER is listed in TABLE I. 
TABLE I

PARAMETERS OF THE BIT-LOADING

\begin{tabular}{|c|c|c|c|c|c|c|c|c|}
\hline \multicolumn{8}{|c|}{ Parameters for bit-loading with modulation gap } \\
\hline BER & $10^{-2}$ & $10^{-3}$ & $10^{-4}$ & $10^{-5}$ & $10^{-6}$ & $10^{-7}$ & $10^{-8}$ & $10^{-9}$ \\
\hline$\Gamma$ (Linear) & 1.80 & 3.18 & 4.61 & 6.07 & 7.53 & 9.02 & 10.50 & 11.99 \\
\hline \multicolumn{8}{|c|}{ Parameters for bit-loading with LA expression } \\
\hline BER & $10^{-2}$ & $10^{-3}$ & $10^{-4}$ & $10^{-5}$ & $10^{-6}$ & $10^{-7}$ & $10^{-8}$ & $10^{-9}$ \\
\hline$A_{1}$ & 2.936 & 3.020 & 3.044 & 3.064 & 3.079 & 3.079 & 3.087 & 3.087 \\
\hline$A_{2}$ & 1.983 & 4.309 & 5.896 & 7.056 & 7.956 & 8.756 & 9.409 & 10.01 \\
\hline
\end{tabular}

TABLE II

RELATIONSHIP BETWEEN REQUIRED SNR AND DESIRED BER IN DIFFERENT QAM MODULATIONS

\begin{tabular}{|c|c|c|c|c|c|c|c|c|}
\hline & \multicolumn{7}{|c|}{ Required SNR (dB) for desired BER } \\
\hline BER & $10^{-2}$ & $10^{-3}$ & $10^{-4}$ & $10^{-5}$ & $10^{-6}$ & $10^{-7}$ & $10^{-8}$ & $10^{-9}$ \\
\hline 2-PSK & 4.3 & 6.8 & 8.4 & 9.6 & 10.5 & 11.3 & 12.0 & 12.6 \\
\hline 4-QAM & 7.3 & 9.8 & 11.4 & 12.6 & 13.5 & 14.3 & 15.0 & 15.6 \\
\hline 8-QAM & 11.9 & 14.4 & 16.1 & 17.3 & 18.3 & 19.1 & 19.7 & 20.3 \\
\hline 16-QAM & 13.9 & 16.5 & 18.2 & 19.4 & 20.4 & 21.2 & 21.9 & 22.5 \\
\hline 32-QAM & 16.9 & 19.6 & 21.3 & 22.5 & 23.5 & 24.3 & 25.0 & 25.6 \\
\hline 64-QAM & 19.8 & 22.6 & 24.3 & 25.6 & 26.6 & 27.4 & 28.1 & 28.7 \\
\hline 128-QAM & 22.7 & 25.5 & 27.3 & 28.6 & 29.6 & 30.4 & 31.1 & 31.6 \\
\hline 256-QAM & 25.4 & 28.4 & 30.2 & 31.5 & 32.5 & 33.3 & 34.0 & 34.6 \\
\hline 512-QAM & 28.3 & 31.3 & 33.1 & 34.4 & 35.5 & 36.3 & 37.0 & 37.6 \\
\hline 1024-QAM & 31.1 & 34.3 & 36.1 & 37.5 & 38.5 & 39.3 & 40.1 & 40.7 \\
\hline
\end{tabular}

\section{B. Bit-loading from Linear Approximation Expression}

In this paper, we propose a new approach to implement bit-loading from a linear approximation of BER-SNR table. The relationship between the BER and SNR can be calculated based on the well-known error probability notation [22]:

$$
\varepsilon_{b}=\mathrm{Q}\left(\sqrt{\frac{d^{2}}{2 N_{0}}}\right),
$$

where $d$ is the distance between two constellation points. $N_{0}$ is the noise power spectral density. Based on Eq. (4), the error probability for square constellation and rectangle constellation QAM are given in [22]. The theoretical derivation of the error probability for 32-QAM with cross constellation map is given in [17]. When 128-QAM and 512-QAM are used with cross constellation maps, the Monte-Carlo simulation is adopted to calculate the required SNR to reach a given BER. For each SNR, we stopped after 200 errors. Therefore for the desired BER at $1 \times 10^{-9}$, we simulated more than $2 \times 10^{11}$ points.

The theoretical results of the abovementioned QAM modulations with the relationships between required SNR and BER performance are listed in TABLE II. It is worth noting that a linear approximation of the SNR can be done for each desired BER. The least squares estimation is used to obtain a linear expression of the relationship between the SNR and the number of allocated bits [23]. The presentation of the LA expression between the allocated bits $b$ and required SNR in different BER cases can be given as:

$$
b=\left[\operatorname{SNR}(d B)-A_{2}\right] / A_{1},
$$

where $\operatorname{SNR}(d B)$ is similar with the definition in Eq. (1). The parameters $A_{1}$ and $A_{2}$ are estimated from the calculated results in TABLE II. A list of the obtained parameters $A_{1}$ and $A_{2}$ for different desired BER is presented in TABLE I. Introducing

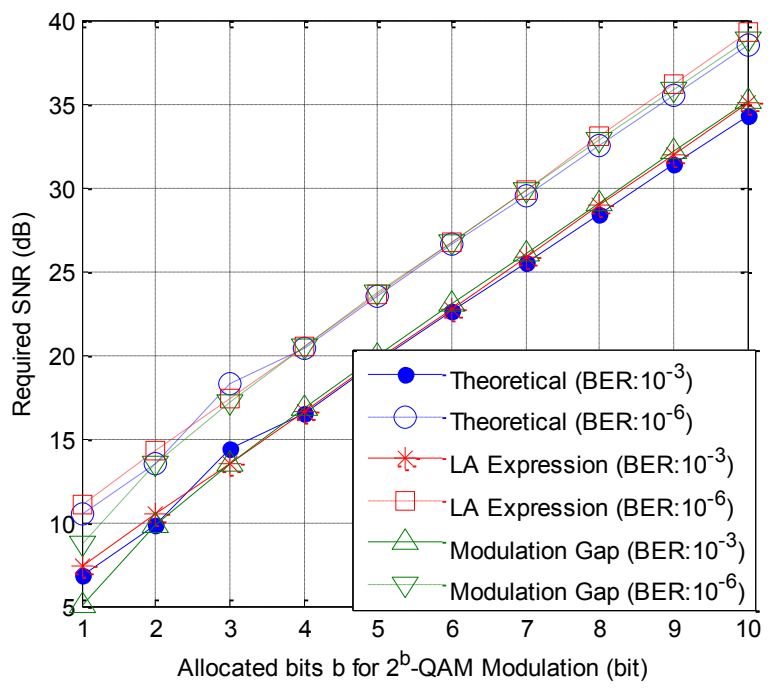

Fig. 2. Relationship of required SNRs for theoretical results, LA expression results and modulation gap results

the parameters in TABLE I and II, the required SNR for theoretical results, modulation gap results from Eq. (1) and LA expression results from Eq. (5) are depicted in Fig. 2. As shown in the figure, both bit-loading algorithms can approach the theoretical required SNR in most of the cases ( $b$ from 2 to 10), except slightly deviation in 8-QAM with rectangle constellation map. However, the modulation gap based results get a significant approximation offset of the SNR for the 2-PSK modulation. This offset of 2-PSK will result in an inaccurate used bandwidth after bit-loading and wasting of the power.

\section{Implementation of Bit-loading in DMT Transmission}

The DMT modulation signal can be expressed as follows:

$$
\begin{gathered}
S(k)=\frac{1}{\sqrt{2 N}} \sum_{n=0}^{2 N-1} \operatorname{Re}\left\{X_{n} \exp \left(j 2 \pi n \frac{k}{2 N}\right)\right\}, \\
k=0,1, \ldots, 2 N-1, \quad X_{2 N-n}=X_{n}^{*},
\end{gathered}
$$

where $S(k)$ is the modulated real-valued DMT signal, which requires $2 N$ points of IFFT [6]. $N$ is total available subcarriers for DMT. The original signal $X_{n}$ follows Hermitian symmetry property in Eq. (6). In DMT modulation, $X_{n}$ is mapped with different $2^{b}$-QAM modulation. After the transmission and equalization, each $X_{n}$ has a $\mathrm{SNR}_{n}$. In the classical bit-loading with modulation gap algorithms, the total available transmission rate for one DMT symbol can be expressed as:

$$
R=\sum_{n=1}^{N} b_{n}=\sum_{n=1}^{N} \log _{2}\left[1+\frac{10^{\left(\mathrm{SNR}_{\mathrm{n}}(d B) / 10\right)}}{\Gamma}\right]
$$

where $\Gamma$ is obtained with pre-calculated parameters in TABLE I. For bit-loading with LA expression, the total available transmission rate for the determinate subcarrier SNR can be obtained with a simpler equation:

$$
R=\sum_{n=1}^{N} b_{n}=\sum_{n=1}^{N}\left[\operatorname{SNR}_{n}(d B)-A_{2}\right] / A_{1},
$$

where $A_{1}$ and $A_{2}$ can also be obtained with pre-calculated 


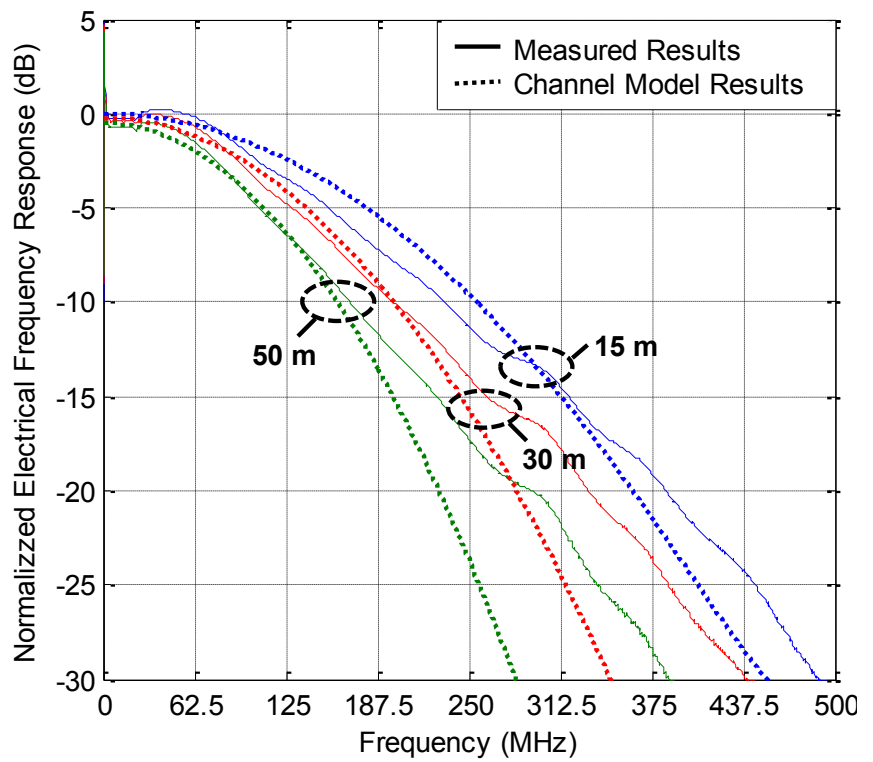

Fig. 3. Normalized frequency responses of SI-POF with different length

parameters in TABLE I.

\section{Performance Bound of Bit-loading ANd Power ALLOCATION FOR DMT TRANSMISSION OVER SI-POF}

\section{A. Introduction of Gaussian Low-pass Channel Model}

The SI-POF channel presents serious modal dispersions that cause a low-pass frequency response. The low-pass channel frequency response of SI-POF channel can be modeled as Gaussian low-pass filter, which is shown as follows [2]:

$$
H(f)=A \cdot e^{-\left(\frac{f}{f_{0}}\right)^{2}}, \quad f_{0}=f_{3 d B} / \sqrt{\ln (2)},
$$

where $A$ is the fiber loss, $f_{3 d B}$ is the $3 \mathrm{~dB}$ bandwidth of the channel frequency response. In a practical SI-POF system, the received signal suffers from various noises and distortions [24]. The non-linear distortion caused noise and quantization noise can be reduced by optimized system setup [24]. Several noises such as the modal noise in the fiber and receiver noise after the auto-gain control (AGC) and amplification are related to the transmitted signal. Therefore the normalized noise power spectral density (PSD) in DMT transmission over SI-POF can be measured by using the channel estimation information obtained from the DMT probing symbols. Then, the discrete presentation of the subcarrier SNR with the Gaussian low-pass channel model can be expressed as:

$$
\operatorname{SNR}_{n}(d B)=10 . \log _{10}\left(\frac{P_{n} \cdot e^{-\left(\frac{f_{n}}{f_{0}}\right)^{2}}}{N_{0} \cdot \Delta f}\right),
$$

where $P_{n}$ is the allocated power at $n^{\text {th }}$ subcarrier, $f_{n}$ is the frequency at $n^{t h}$ subcarrier, $N_{0}$ is the normalized noise PSD, $\Delta f$ is the frequency spacing between subcarriers. The comparisons of the measured and Gaussian low-pass filter modeled channel frequency response of SI-POF channels are shown in Fig. 3. We can note that for low frequencies, the curves match well. For high frequencies, as the signals are seriously attenuated, the mismatches between real and channel model curves are not significant to the overall system. The detailed experimental setups will be presented in Section V.

\section{B. Maximization of the Bit Rate in Bit-loading with Modulation Gap}

In this part, we use the water-filling to optimize the power allocation $P_{n}$ for each subcarrier of a DMT transmission over the Gaussian low-pass channel model. Using the $\mathrm{SNR}_{n}$ provided by Eq. (10) and total transmission rate in Eq. (7), the expression of the transmission rate in a DMT system with modulation gap based bit-loading is expressed as:

$$
R=\sum_{n=1}^{N_{\max }} \log _{2}\left(1+\frac{P_{n}}{\Gamma \cdot N_{0} \cdot \Delta f} \cdot e^{-\left(\frac{f_{n}}{f_{0}}\right)^{2}}\right) .
$$

under the constrained condition of total limited power:

$$
\sum_{n=1}^{N_{\max }} P_{n}=P_{t}
$$

where $N_{\max }$ is the number of maximal used subcarriers, $P_{t}$ is the constrained total power. The Lagrange multiplier [25] can be employed to solve the optimization problem in Eq. (11) and Eq. (12). Introducing the Lagrange multiplier, we can obtain the optimal power allocation in bit-loading with modulation gap. The detailed derivation from Eq. (11) to Eq. (16) is presented in APPENDIX A. The obtained optimal power for each subcarrier after the water-filling optimization is given as:

$$
P_{n}=\frac{P_{t}+\Delta}{N_{\max }}-\Gamma \cdot N_{0} \cdot \Delta f \cdot e^{\left(\frac{f_{n}}{f_{0}}\right)^{2}},
$$

where $\Delta$ is a constant value shown as:

$$
\Delta=\Gamma \cdot N_{0} \cdot \Delta f \cdot \frac{\sqrt{\pi}}{2} \cdot N_{\max } \cdot \frac{f_{0}}{f_{\max }} \cdot\left[\operatorname{erfi}\left(\frac{f_{\max }}{f_{0}}\right)\right],
$$

$f_{\max }$ is the maximal used bandwidth, $\operatorname{erfi}(\quad)$ is the imaginary error function, as detailed in APPENDIX A. The calculated maximal bit rate for a DMT symbol is shown as:

$$
\begin{aligned}
R= & N_{\max } \cdot \log _{2}\left(\frac{\left(P_{t}+\Delta\right)}{\Gamma \cdot N_{0} \cdot \Delta f \cdot N_{\max }}\right)-\frac{\Delta f^{2}}{\ln 2 \cdot N^{2} \cdot{f_{0}}^{2}} . \\
& \frac{1}{6} \cdot N_{\max } \cdot\left(N_{\max }+1\right) \cdot\left(2 N_{\max }+1\right) .
\end{aligned}
$$

The maximal used bandwidth is:

$$
\begin{gathered}
N_{\max }=\max (n) \text { s.t. } \\
\frac{P_{t}+\Delta}{n}-\Gamma \cdot N_{0} \cdot \Delta f \cdot e^{\left(\frac{f_{n}}{f_{0}}\right)^{2}} \geq 0 .
\end{gathered}
$$

In order to reach optimal bit-loading performance, the power allocations in Eq. (13) are adjusted according to the overall channel response owning to the Gaussian low-pass channel. Therefore, the transmitted powers allocated to each subcarrier are pre-emphasized with the results in Eq. (13). Nevertheless in practical systems, the pre-emphasizing with the theoretically optimized result in Eq. (13) is not usually considered due to its 
complexity. The constant power allocation with iterative process is often used. This technique will be discussed in Section IV.

\section{Maximization of the Bit Rate in Bit-loading with LA Expression}

For bit-loading with LA expression, using $\mathrm{SNR}_{n}$ provided by Eq. (10) and total transmission rate in Eq. (8), the expression of the transmission rate in the DMT transmission over SI-POF channel is expressed as follows:

$$
R=\sum_{n=1}^{N_{\max }}\left(10 \cdot \log _{10}\left(\frac{P_{n}}{N_{0} \cdot \Delta f} \cdot e^{-\left(\frac{f_{n}}{f_{0}}\right)^{2}}\right)-A_{2}\right) / A_{1},
$$

under the constrained condition

$$
\sum_{n=1}^{N_{\max }} P_{n}=P_{t}
$$

Introducing the Lagrange multiplier for the optimization, we can obtain the optimal power allocation with LA expression. The detailed derivation from Eq. (17) to Eq. (21) is presented in APPENDIX B. The optimal power allocation in LA expression based bit-loading is expressed as:

$$
P_{n}=\frac{P_{t}}{N_{\max }}
$$

and the maximal bit rate for a DMT symbol is:

$$
\begin{aligned}
R= & N_{\max } \cdot\left[\frac{10}{A_{1}} \cdot \log _{10}\left(\frac{P_{t}}{N_{\max } \cdot N_{0} \cdot \Delta f}\right)-\frac{A_{2}}{A_{1}}\right] \\
& -\frac{10 \cdot \Delta f^{2}}{A_{1} \cdot \ln 10 \cdot N^{2} \cdot f_{0}{ }^{2}} \cdot \frac{1}{6} \cdot N_{\max } \cdot\left(N_{\max }+1\right) \\
& \cdot\left(2 N_{\max }+1\right) .
\end{aligned}
$$

The maximal used bandwidth is:

$$
\begin{gathered}
N_{\max }=\max (n) \quad \text { s.t. } \\
10 \cdot \log _{10}\left(\frac{P_{n}}{N_{0} \cdot \Delta f} \cdot e^{-\left(\frac{f_{n}}{f_{0}}\right)^{2}}\right)-A_{2} \geq 0 .
\end{gathered}
$$

It is worth noting that in Eq. (19), while taking into account water-filling optimization for bit-loading with LA expression, the maximal transmission rate for the Gaussian low-pass channel can be achieved by constant power allocation. Therefore in order to reach the maximal transmission rate, the pre-emphasizing of the power for each subcarrier is not required. This can reduce the implementation complexity compared to the modulation gap based bit-loading.

\section{BIT-LOADING AND POWER ALLOCATION FOR DMT TRANSMISSION OVER SI-POF}

In Section III, the theoretical transmission rate was optimized by the aforementioned derivations, but the practical approach of the bit-loading for a real DMT transmission system is slightly different. In a real system, the water filling algorithm is not carried out due to its high complexity. Therefore a constant power allocation without the pre-emphasizing of the power for each subcarrier is usually adopted [10] and an iterative process is used to obtain the sub-optimal performance [14].

In addition, the fractional bits modulation is not practical in a regular $2^{b}$-order QAM. Hence the practical allocated bit $b_{n}^{\prime}$ for each subcarrier of the DMT follows the formula as:

$$
b_{n}^{\prime}=\operatorname{round}\left[\log _{2}\left(1+\frac{10^{\left(\left(\operatorname{SNR}_{\mathrm{n}}(d B)-\gamma_{\text {margin }}\right) / 10\right)}}{\Gamma}\right)\right]
$$

for bit-loading with modulation gap, and

$$
b_{n}^{\prime}=\operatorname{round}\left[\left(\operatorname{SNR}_{n}(d B)-\gamma_{\text {margin }}-\mathrm{A}_{2}\right) / A_{1}\right]
$$

for bit-loading with LA expression. The $\gamma_{\text {margin }}$ is the current system performance margin for the iterative process.

Based on Chow algorithm, the iterative process of bit-loading for both cases is detailed as follows [14]:

Step 1: Estimate the $\operatorname{SNR}_{n}(d B)$ of each subcarrier. This process can be implemented by the transmission of several 4-QAM probing DMT symbols. After the transmission, the SNR of each subcarrier can be estimated. As the constant power allocation is adopted, the $P_{n}$ is 1 for each subcarrier and $\gamma_{\text {margin }}$ is 0 .

Step 2: Allocate bits for each subcarrier using Eq. (22) for the classical modulation gap based bit-loading or Eq. (23) for the proposed algorithms. According to the different desired BER levels, the required parameters $\Gamma, A_{1}$ and $\mathrm{A}_{2}$ are listed in TABLE I. After bit-loading, some subcarriers are not allocated due to their low SNR, which lead to an adjusted number of used subcarriers. Here we consider that $N_{\max }$ is the number of used subcarriers in our SI-POF channel. Then $\gamma_{\text {margin }}$ should be changed in order to obtain the achievable transmission rate with the new value of $N_{\max }$. Therefore iterative process is required to obtain the optimal $N_{\text {max }}$ and $\gamma_{\text {margin }}$.

Step 3: Calculate the used power by $N_{\max }: P_{t}^{\prime}=P_{n} \cdot N_{\max }$. In the first iteration, the original used power is $P_{t}=P_{n} \cdot N$. The system performance margin can be refreshed as:

$$
\gamma_{\text {margin }}^{(\text {new })}=\gamma_{\text {margin }}^{(\text {old })}+10 \cdot \log _{10}\left(P_{t}^{\prime} / P_{t}\right) \text {. }
$$

Then we refresh $P_{t}$ with $P_{t}=P_{t}^{\prime}$ and go to Step 2 for the next iteration. The maximal transmission rate $R$ with the optimal number of used subcarriers $N_{\max }$ can be sub-optimally achieved after several iterations between Step 2 and Step 3 .

As being mentioned in Step 2, for practical implementations, the difference between the proposed LA expression bit-loading and classical modulation gap bit-loading algorithms is only the use of Eq. (23) instead of Eq. (22). Bit-loading can be easily achieved by this iterative process.

It is worth noting that the rounding functions in Eq. (22) and Eq. (23) adjust the actual required power for each subcarrier. In order to estimate the accurate BER for each subcarrier, the power of the $n^{\text {th }}$ subcarrier should be slightly adjusted according the actual required SNR. As the $b_{n}^{\prime}$ is known, the actual required $\operatorname{SNR}_{n}^{\prime}(d B)$ for QAM can be obtained. The actual required power $P_{n}^{\prime}$ is adjusted with following equation:

$$
P_{n}^{\prime}=P_{n} \cdot 10\left(\left(\operatorname{SNR}_{n}(d B)-\mathrm{SNR}_{n}^{\prime}(d B)\right) / 10\right) .
$$

Although the constant power allocation is employed in the iterative process, after the process in Eq. (25), the power 


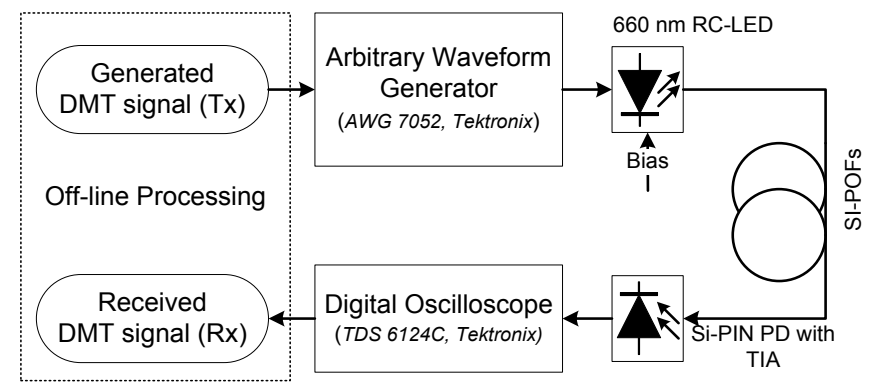

Fig. 4. Experimental Setups

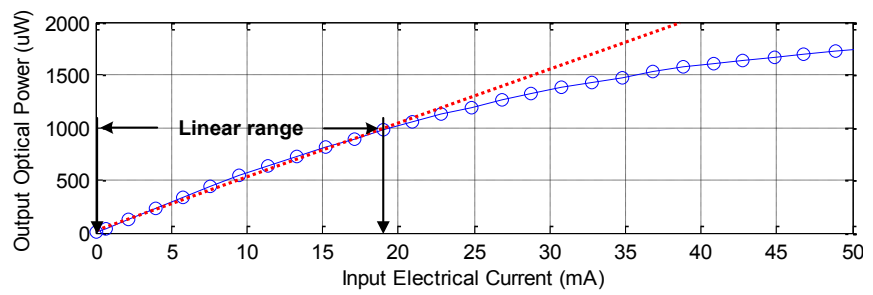

Fig. 5. Measured RCLED electrical-optical curve

allocated for each subcarrier is variable due to the different required power for each subcarrier. However, comparing Eq. (25) with Eq. (13), it is clear that this variable power allocation is different from the modulation gap based bit-loading employing water-filling. As being mentioned in Section III.B, Eq. (13) requires power pre-emphasizing for each subcarrier according the overall channel response.

\section{MEASUREMENT OF SI-POF CHANNEL MODEL}

\section{A. Experimental Setups}

We built an experimental system to measure the SI-POF channel response. SI-POFs (ESKA ${ }^{\mathrm{TM}}$ Mega, Mitsubishi) with different lengths are measured and modeled. The experimental setups are depicted in Fig. 4. The testing signal is transmitted from the output of digital-to-analog convertor (DAC). A biasing current is added to bias the output signal. A commercial available RCLED (FC300R-120 ${ }^{\mathrm{TM}}$, Firecomms) with $660 \mathrm{~nm}$ wavelength and measured $135 \mathrm{MHz} 3 \mathrm{~dB}$ bandwidth is used for the optical source. Because our proposed derivation in Section III is based on Gaussian low-pass filter channel model without the consideration of channel non-linearity, the real channel model parameters should be measured without non-linearity distortions of the RCLED [26]. In case of the RCLED non-linearity is introduced to the system, the power allocations in subcarriers at low frequencies are more complicate than the theoretical results in Section III [24][27]. Therefore RCLED working current should be limited in a linear range. Furthermore, due to the AGC functions in the receiver, increasing RCLED working current to non-linear range can hardly benefit improvement in very short distance transmissions (Less than $50 \mathrm{~m}$ ). A static relationship of the input electrical current and output optical power of the experimental RCLED is measured and depicted in Fig. 5. As shown in the figure, the linear working range of the RCLED is around 0 to $20 \mathrm{~mA}$. Thus a $10 \mathrm{~mA}$ biasing current is selected in the experiments. The biased signal is directly fed into the RCLED and the measured output optical power is $-2.5 \mathrm{dBm}$. SI-POFs with different length are installed. They were cut with cutter blade without polishing. After the transmission over
TABLE III

PARAMETERS OF SI-POF CHANNEL MODELS

\begin{tabular}{|c|c|c|c|c|c|}
\hline Length & $15 \mathrm{~m}$ & $30 \mathrm{~m}$ & $50 \mathrm{~m}$ & $75 \mathrm{~m}$ & $100 \mathrm{~m}$ \\
\hline$f_{3 \mathrm{~dB}}$ & $130 \mathrm{MHz}$ & $110 \mathrm{MHz}$ & $90 \mathrm{MHz}$ & $65 \mathrm{MHz}$ & $55 \mathrm{MHz}$ \\
\hline Noise PSD & -114.2 & -114.0 & -113.7 & -109.8 & -102.6 \\
$N_{0}$ & $\mathrm{~dB} / \mathrm{Hz}$ & $\mathrm{dB} / \mathrm{Hz}$ & $\mathrm{dB} / \mathrm{Hz}$ & $\mathrm{dB} / \mathrm{Hz}$ & $\mathrm{dB} / \mathrm{Hz}$ \\
\hline
\end{tabular}

SI-POF, the received optical powers are respectively measured at $-5.4 \mathrm{dBm},-8.8 \mathrm{dBm},-10.9 \mathrm{dBm},-16.9 \mathrm{dBm}$ and $-21.5 \mathrm{dBm}$ for a $15 \mathrm{~m}, 30 \mathrm{~m}, 50 \mathrm{~m}, 75 \mathrm{~m}$ and $100 \mathrm{~m}$ transmission. The loss of the optical power is mainly due to the POF attenuation. The insertion loss by cutter blade without polishing causes 1 to $2 \mathrm{~dB}$ loss [28]. We use an integrated receiver component (FC1000D-120 ${ }^{\mathrm{TM}}$, Firecomms), which is a Si-PIN photo-detector (PD) with a $700 \mu \mathrm{m}$ diameter active area followed by a trans-impedance amplifier (TIA) and combined with an AGC. This receiver has $625 \mathrm{MHz} 3 \mathrm{~dB}$ bandwidth and AGC range from $-27 \mathrm{dBm}$ to $1 \mathrm{dBm}$. Finally, the amplified electrical signal is captured by the analog-to-digital convertor (ADC).

\section{B. Measured Channel Models}

The measured $3 \mathrm{~dB}$ transmission bandwidth and normalized noise PSD $N_{0}$ are listed in the TABLE III. As the power of each subcarrier $P_{n}$ is normalized to 1 , the $N_{0}$ is expressed as $\mathrm{dB} / \mathrm{Hz}$. As shown in TABLE III, when SI-POF length is less than $50 \mathrm{~m}$, the variation of the measured noise PSD is slight, and when SI-POF is longer than $50 \mathrm{~m}$, the noise PSD relatively increases because Si-PIN diode and TIA become the dominant noise sources.

\section{Simulation Results}

\section{A. Results of Theoretical Performance Bounds}

With the help of the channel $3 \mathrm{~dB}$ bandwidth $f_{3 d B}$ and the noise PSD $N_{0}$, the maximal transmission rate can be theoretically calculated by Eq. (15) and Eq. (20) in Section III. Meanwhile, the maximal used bandwidth can be calculated by Eq. (16) and Eq. (21). The number of subcarriers $N$ is selected as 512 for the evaluation. The frequency spacing $\Delta f$ of each subcarrier is $1 \mathrm{MHz}$. Four desired BER levels from $1 \cdot 10^{-3}$ to $1 \cdot 10^{-6}$ are simulated. The available net transmission rate $R$ and optimal used bandwidth $f_{\max }$ of SI-POF systems with different lengths and different desired BER are mathematically obtained in TABLE IV. As shown in the table, in theoretical computations, both LA expression based bit-loading and modulation gap based bit-loading can reach similar maximal transmission rates and used bandwidth after optimal power allocation.

However, it is worth noting that employing water-filling, the optimal power allocation computation for modulation gap based bit-loading in Eq. (13) is much more complex than that in the proposed LA expression based bit-loading in Eq. (19).

\section{B. Results of Practical Transmission Rate}

In most of the practical applications, Chow algorithm with iterative process is used for bit allocation in DMT systems [9][20]. We introduce the practical bit-loading employing the iterative process in Section IV. Being different from the theoretical optimizations in the last part, the constant power 
TABLE IV

\begin{tabular}{|c|c|c|c|c|c|}
\hline \multicolumn{6}{|c|}{ IMULATION RESULTS OF THEORETICAL PERFORMANCE BOUNI } \\
\hline \multirow[b]{2}{*}{$\begin{array}{l}\text { Desired } \\
\text { BER }\end{array}$} & \multirow[b]{2}{*}{$\begin{array}{l}\text { SI-POF } \\
\text { Length }\end{array}$} & \multicolumn{2}{|c|}{$\begin{array}{l}\text { Bit-loading with } \\
\text { LA Expression }\end{array}$} & \multicolumn{2}{|c|}{$\begin{array}{l}\text { Bit-loading with } \\
\text { Modulation Gap }\end{array}$} \\
\hline & & $\begin{array}{c}\text { Bit } \\
\text { Rate } \\
(\mathrm{Mbps})\end{array}$ & $\begin{array}{c}\text { Used } \\
\text { Bd. } \\
\text { (MHz) }\end{array}$ & $\begin{array}{c}\text { Bit } \\
\text { Rate } \\
(\mathrm{Mbps})\end{array}$ & $\begin{array}{c}\text { Used } \\
\text { Bd. } \\
(\mathrm{MHz})\end{array}$ \\
\hline \multirow{5}{*}{$10^{-3}$} & $15 \mathrm{~m}$ & 1967 & 368 & 1945 & 366 \\
\hline & $30 \mathrm{~m}$ & 1715 & 314 & 1694 & 313 \\
\hline & $50 \mathrm{~m}$ & 1447 & 260 & 1429 & 259 \\
\hline & $75 \mathrm{~m}$ & 905 & 179 & 896 & 178 \\
\hline & $100 \mathrm{~m}$ & 494 & 130 & 494 & 131 \\
\hline \multirow{5}{*}{$10^{-4}$} & $15 \mathrm{~m}$ & 1778 & 357 & 1773 & 355 \\
\hline & $30 \mathrm{~m}$ & 1552 & 305 & 1546 & 303 \\
\hline & $50 \mathrm{~m}$ & 1313 & 252 & 1307 & 251 \\
\hline & $75 \mathrm{~m}$ & 815 & 173 & 813 & 172 \\
\hline & $100 \mathrm{~m}$ & 430 & 125 & 437 & 125 \\
\hline \multirow{5}{*}{$10^{-5}$} & $15 \mathrm{~m}$ & 1647 & 348 & 1650 & 347 \\
\hline & $30 \mathrm{~m}$ & 1439 & 298 & 1441 & 296 \\
\hline & $50 \mathrm{~m}$ & 1218 & 247 & 1219 & 245 \\
\hline & $75 \mathrm{~m}$ & 751 & 169 & 754 & 168 \\
\hline & $100 \mathrm{~m}$ & 387 & 121 & 396 & 121 \\
\hline \multirow{5}{*}{$10^{-6}$} & $15 \mathrm{~m}$ & 1546 & 342 & 1555 & 340 \\
\hline & $30 \mathrm{~m}$ & 1353 & 292 & 1360 & 291 \\
\hline & $50 \mathrm{~m}$ & 1147 & 242 & 1152 & 241 \\
\hline & $75 \mathrm{~m}$ & 704 & 165 & 709 & 165 \\
\hline & $100 \mathrm{~m}$ & 354 & 118 & 366 & 118 \\
\hline
\end{tabular}

allocation is performed in the bit-loading, without the pre-emphasizing of the power for each subcarrier.

The simulations have been implemented in Matlab ${ }^{\mathrm{TM}}$. Based on the experimental optimizations, DMT with 512 subcarriers is considered as an optimal selection in the SI-POF system, taking into account performance and complexity [24]. Therefore 512 DMT subcarriers are generated to implement bit-loading algorithms. A total frequency range of $512 \mathrm{MHz}$ is set with a $1 \mathrm{MHz}$ subcarrier frequency spacing. The results of the $S N R_{n}$ in different SI-POF channel models are calculated from the channel models in TABLE III, which are depicted in Fig. 6. Four desired BER levels from $1 \cdot 10^{-3}$ to $1 \cdot 10^{-6}$ are simulated.

The simulation results of practical transmission rates are listed in TABLE V. As shown in the table, in all of the practical

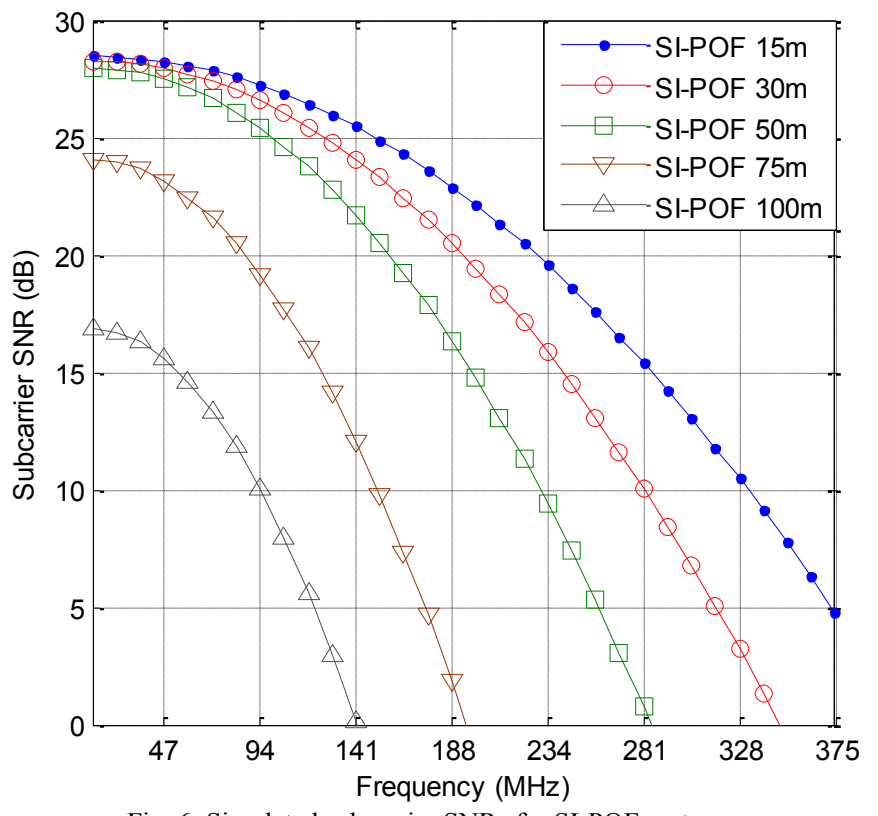

Fig. 6. Simulated subcarrier SNRs for SI-POF systems
TABLE V

\begin{tabular}{|c|c|c|c|c|c|c|}
\hline \multirow[b]{2}{*}{$\begin{array}{c}\text { Desired } \\
\text { BER }\end{array}$} & \multirow[b]{2}{*}{$\begin{array}{l}\text { SI-POF } \\
\text { Length }\end{array}$} & \multicolumn{2}{|c|}{$\begin{array}{l}\text { Bit-loading with } \\
\text { LA Expression }\end{array}$} & \multicolumn{2}{|c|}{$\begin{array}{l}\text { Bit-loading with } \\
\text { Modulation Gap }\end{array}$} & \multirow{2}{*}{$\begin{array}{c}\text { Data } \\
\text { Rate Gain } \\
\text { in LA } \\
\text { Expression }\end{array}$} \\
\hline & & $\begin{array}{c}\text { Bit } \\
\text { Rate } \\
\text { (Mbps) }\end{array}$ & $\begin{array}{l}\text { Used } \\
\text { Bd. } \\
(\mathrm{MHz})\end{array}$ & $\begin{array}{c}\text { Bit } \\
\text { Rate } \\
\text { (Mbps) }\end{array}$ & $\begin{array}{l}\text { Used } \\
\text { Bd. } \\
(\mathrm{MHz})\end{array}$ & \\
\hline \multirow{5}{*}{$10^{-3}$} & $15 \mathrm{~m}$ & 1958 & 356 & 1886 & 382 & $3.8 \%$ \\
\hline & $30 \mathrm{~m}$ & 1705 & 305 & 1647 & 330 & $3.5 \%$ \\
\hline & $50 \mathrm{~m}$ & 1437 & 253 & 1391 & 273 & $3.3 \%$ \\
\hline & $75 \mathrm{~m}$ & 896 & 173 & 864 & 189 & $3.7 \%$ \\
\hline & $100 \mathrm{~m}$ & 486 & 124 & 459 & 139 & $5.9 \%$ \\
\hline \multirow{5}{*}{$10^{-4}$} & $15 \mathrm{~m}$ & 1764 & 345 & 1703 & 375 & $3.6 \%$ \\
\hline & $30 \mathrm{~m}$ & 1541 & 294 & 1484 & 321 & $3.8 \%$ \\
\hline & $50 \mathrm{~m}$ & 1303 & 243 & 1252 & 266 & $4.1 \%$ \\
\hline & $75 \mathrm{~m}$ & 807 & 167 & 775 & 182 & $4.1 \%$ \\
\hline & $100 \mathrm{~m}$ & 425 & 119 & 397 & 132 & $7.1 \%$ \\
\hline \multirow{5}{*}{$10^{-5}$} & $15 \mathrm{~m}$ & 1635 & 335 & 1569 & 365 & $4.2 \%$ \\
\hline & $30 \mathrm{~m}$ & 1427 & 288 & 1376 & 312 & $3.7 \%$ \\
\hline & $50 \mathrm{~m}$ & 1204 & 239 & 1166 & 259 & $3.3 \%$ \\
\hline & $75 \mathrm{~m}$ & 742 & 162 & 709 & 178 & $4.7 \%$ \\
\hline & $100 \mathrm{~m}$ & 382 & 114 & 355 & 129 & $7.6 \%$ \\
\hline \multirow{5}{*}{$10^{-6}$} & $15 \mathrm{~m}$ & 1534 & 328 & 1465 & 360 & $4.7 \%$ \\
\hline & $30 \mathrm{~m}$ & 1342 & 281 & 1287 & 307 & $4.3 \%$ \\
\hline & $50 \mathrm{~m}$ & 1137 & 233 & 1094 & 253 & $3.9 \%$ \\
\hline & $75 \mathrm{~m}$ & 694 & 159 & 665 & 174 & $4.4 \%$ \\
\hline & $100 \mathrm{~m}$ & 349 & 111 & 326 & 124 & $7.1 \%$ \\
\hline
\end{tabular}

implementations, bit-loading with LA expression can achieve higher transmission rates than bit-loading with modulation gap.

Moreover, in all of the simulation results, the transmission rate and used bandwidth of bit-loading with LA expression are slightly less than the theoretically calculated optimal results. This performance degradation between the theoretical and practical system is mostly due to the round function of the integral number of the allocated bits in Eq. (23), especially for the 2-PSK. It is easy to understand that the subcarriers with theoretically calculated $b_{n}$ less than 0.5 will be set as null in a practical system, which reduce the used bandwidth and the transmission rate.

Furthermore, in the proposed bit-loading approach with LA expression, based on the water-filling theorem in Section III, the optimum energy of each subcarrier in Gaussian low-pass

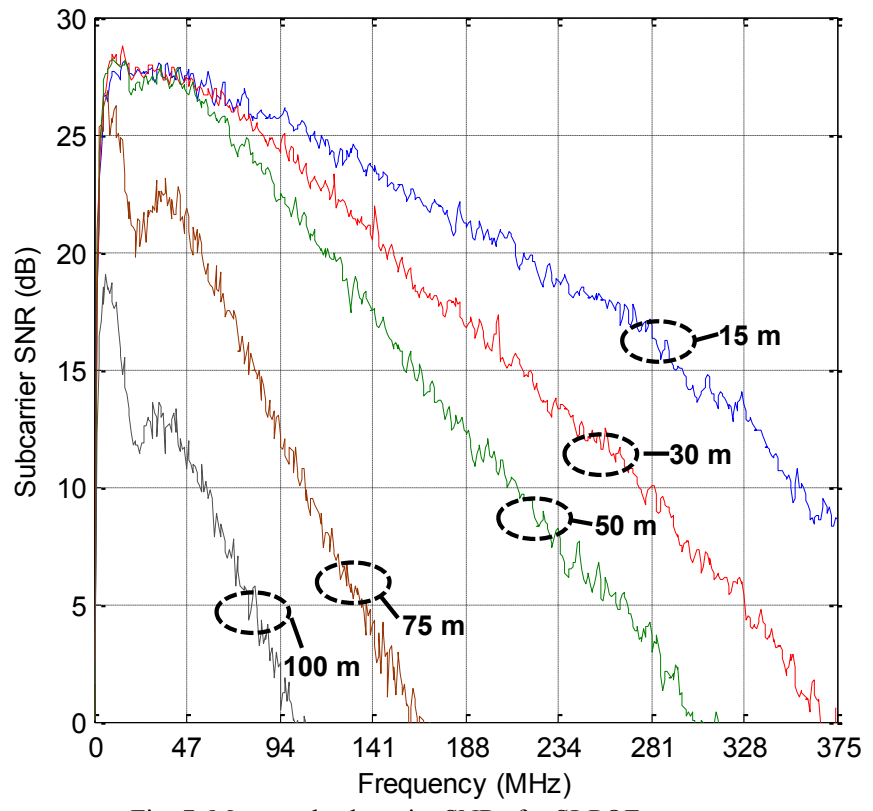

Fig. 7. Measured subcarrier SNRs for SI-POF systems 


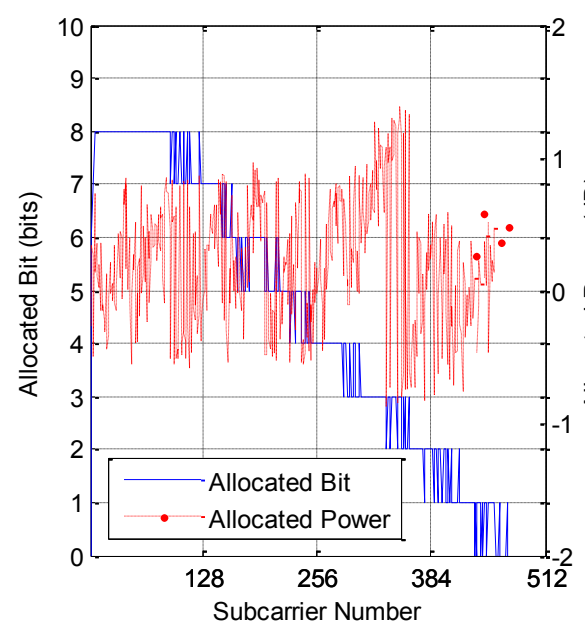

(a) Bit-loading with LA expression

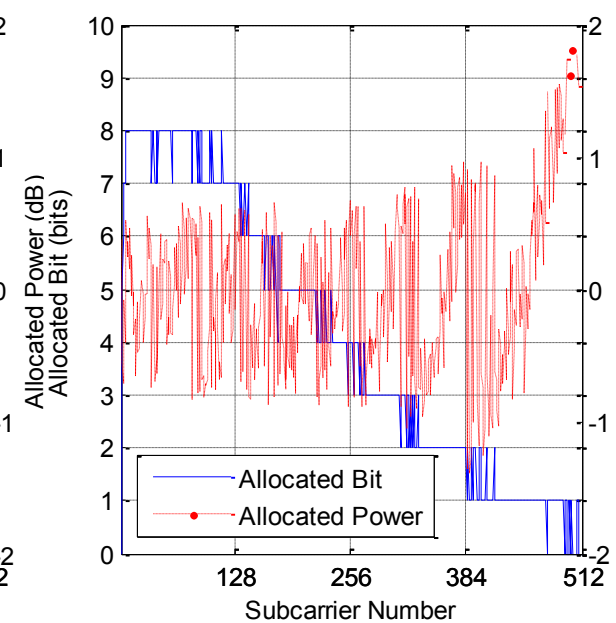

(b) Bit-loading with modulation gap

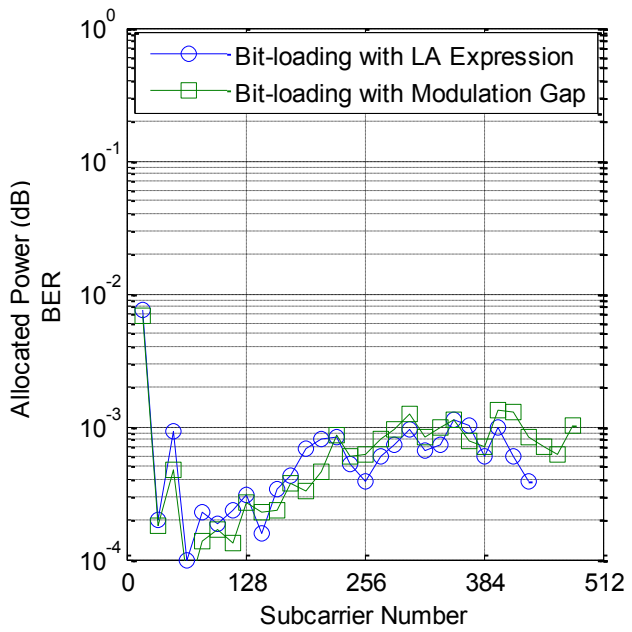

(c) Measured subcarrier BER in receiver

Fig. 8. Illustrations of DMT Transmission over $50 \mathrm{~m}$ SI-POF with different bit-loading approach

TABLE VI

TRANSMISSION RATE IN REAL SI-POF CHANNELS (BER: $1.0 \times 10^{-3}$ )

\begin{tabular}{|c|c|c|c|c|c|c|}
\hline \multicolumn{3}{|c|}{$\begin{array}{l}\text { Bit-loading with } \\
\text { LA Expression }\end{array}$} & \multicolumn{3}{|c|}{$\begin{array}{l}\text { Bit-loading with } \\
\text { Modulation Gap }\end{array}$} & \multirow{2}{*}{$\begin{array}{c}\text { Data } \\
\text { Rate Gain } \\
\text { in LA } \\
\text { Expression }\end{array}$} \\
\hline $\begin{array}{l}\text { SI-POF } \\
\text { length }\end{array}$ & $\begin{array}{c}\text { Bit } \\
\text { Rate } \\
(\mathrm{Mbps})\end{array}$ & $\begin{array}{c}\text { Used } \\
\text { Bd. } \\
\text { (MHz) }\end{array}$ & $\begin{array}{l}\text { SI-POF } \\
\text { length }\end{array}$ & $\begin{array}{c}\text { Bit } \\
\text { Rate } \\
\text { (Mbps) }\end{array}$ & $\begin{array}{l}\text { Used } \\
\text { Bd. } \\
\text { (MHz) }\end{array}$ & \\
\hline $15 \mathrm{~m}$ & 1966 & 375 & $15 \mathrm{~m}$ & 1912 & 375 & $2.8 \%$ \\
\hline $30 \mathrm{~m}$ & 1551 & 328 & $30 \mathrm{~m}$ & 1483 & 355 & $4.6 \%$ \\
\hline $50 \mathrm{~m}$ & 1279 & 275 & $50 \mathrm{~m}$ & 1219 & 298 & $4.9 \%$ \\
\hline $75 \mathrm{~m}$ & 632 & 149 & $75 \mathrm{~m}$ & 593 & 168 & $6.6 \%$ \\
\hline $100 \mathrm{~m}$ & 306 & 100 & $100 \mathrm{~m}$ & 277 & 110 & $10.4 \%$ \\
\hline
\end{tabular}

POF channel model is just the uniformed power, which is derived in Eq. (19). Therefore it is very simple to implement a practical bit-loading with LA expression to approach the optimal channel capacity. However in bit-loading with modulation gap, it is evident that performances have the degradations when sub-optimal Chow algorithms are used without water-filling. Therefore compared to the classical modulation gap based bit-loading, our proposed LA expression based bit-loading can obtain data rate gain in a practical usage, which is calculated in the last column in TABLE V.

Finally, it is worth noting that when the SI-POF transmission length is $100 \mathrm{~m}$, bit-loading with modulation gap data rates degrades more. This performance degradation can be explained from Fig. 2, the modulation gap based bit-loading has worse SNR approximations at low SNR. It is clear that after $100 \mathrm{~m}$ transmission, the SNR is significantly reduced due to the fiber attenuations.

\section{EXPERIMENTAL VERIFICATIONS}

In this section, we setup a real experimental system in order to verify the simulated results in the previous section. The optical experimental setup is the same as the mentioned setups in Fig. 4 and Section V. We initially perform the Step 1 described in Section IV to obtain the SNR on each subcarrier. A DMT system with 512 subcarriers is generated with the 4-QAM probing signals for each subcarrier. After the transmission over real SI-POF channels, the measured results of $\mathrm{SNR}_{n}$ are depicted in Fig. 7. Bit-loading algorithms are implemented by iterating Step 2 and Step 3 in Section IV.

The desired BER of $1 \times 10^{-3}$ is selected for the real transmissions. The achieved transmission rates based on different bit-loading approach are listed in TABLE VI. Similar to the simulated results in TABLE V, the obtained transmission rates with LA expression based bit-loading outperform those with modulation gap based bit-loading, especially for $100 \mathrm{~m}$ SI-POF transmission.

Finally, we demonstrate the detailed experimental setup and results for $50 \mathrm{~m}$ SI-POF transmission system. The transmitted signals are pre-generated in Matlab ${ }^{\mathrm{TM}}$. The allocated bits and power for each subcarrier with LA expression based bit-loading and modulation gap based bit-loading are shown in Fig. 8(a) and Fig. 8(b). The first subcarrier of the DMT symbol is set to null. DAC works at 1.2 GSamples/s. Clipping is not implemented on the DMT signals at the transmitter side. The RCLED works with a $10 \mathrm{~mA}$ biasing in order to avoid the non-linear distortion. After the transmission over $50 \mathrm{~m}$ SI-POF, the received signal is detected by Si-PIN PD and captured by ADC with a sampling rate of 10 GSamples/s. 840 DMT symbols are transmitted, the subcarrier BERs are measured and depicted in Fig. 8(c). The total BER is $7.89 \times 10^{-4}$ for LA expression based bit-loading system, and $7.36 \times 10^{-4}$ for modulation gap based bit-loading system.

As shown in Fig. 8(a) and Fig. 8(b), the used bandwidth of the bit-loading with modulation gap is wider than that of LA expression. Therefore more power is allocated in the last high frequency subcarriers. This power is more efficiently allocated to the subcarriers with better SNR in LA expression.

\section{CONCLUSION}

In this paper, a new bit-loading algorithm with LA expression for maximizing DMT transmission rate for IM/DD optical fiber communication systems is proposed and investigated. The parameters of linear approximation function are estimated by least square estimation of the exact SNR table. Compared to traditional modulation gap based bit-loading, the proposed LA expression based bit-loading provides a better SNR approximation in order to approach the channel capacity. 
The performance bounds of the DMT transmission over Gaussian low-pass channel models are derived. Employing water-filling, the theoretical calculations show that LA expression based bit-loading can optimally reach the maximal transmission rate by constant power allocation. In contrast, the maximal transmission rate in modulation gap based bit-loading system should be achieved by pre-emphasizing power for each subcarrier in terms of water filling from channel response, which increases the complexity for a practical usage.

Based on the Gaussian low-pass channel model, SI-POF transmission systems with different fiber length are modeled for simulations. The theoretical simulations in different fiber length and desired BER show that DMT system after LA expression based bit-loading can achieve similar performance bound than those after the modulation gap based bit-loading with power pre-emphasizing for each subcarrier according the overall channel response. Furthermore, in practical system with iterative process, bit-loading with LA expression and constant power allocation approach the maximal performance bounds. Meanwhile, the modulation gap based bit-loading degrades due to the constant power allocation. Therefore it can be concluded that our proposed LA expression based bit-loading is simpler and more efficient than classical modulation gap based bit-loading.

Finally, experimental verifications are implemented for the proposed LA expression base bit-loading. Compared to the traditional modulation gap based bit-loading system, transmission rate can be improved by $3 \%$ to $10 \%$ when transmission distance increases. In the investigation of an experimental DMT transmission over $50 \mathrm{~m}$ SI-POF, with the same obtained BER level, the DMT transmission rate in LA expression based bit-loading system outperforms by $\%$ than that of classical system and even by $10 \%$ for a $100 \mathrm{~m}$ POF length.

For future works, this LA expression based bit-loading can be easily adapted to other modulations with different constellation maps and could take into account the channel coding, which can further enhance the system performance in order to approach the Shannon capacity. Moreover, the proposed technique gives a simpler and more precise approach for the bit-loading and power allocation in DMT transmissions, which can be easily implemented to other optical OFDM systems in order to improve the spectral efficiency.

\section{APPENDIX A}

Maximization of the Bit-Rate with Modulation Gap

The problem of the optimization is mentioned as follows:

$$
\operatorname{Max} .\left\{\sum_{n=1}^{N_{\max }} \log _{2}\left(1+\frac{P_{n}}{\Gamma \cdot N_{0} \cdot \Delta f} \cdot e^{-\left(\frac{f_{n}}{f_{0}}\right)^{2}}\right)\right\},
$$

under the constrained condition:

$$
\sum_{n=1}^{N_{\max }} P_{n}=P_{t}
$$

Introducing variable $\lambda$ for the Lagrange multiplier,

$$
\begin{aligned}
F(\underbrace{P_{n}}_{n=1,2 \ldots, N_{\max }}, \lambda)= & \left\{\sum_{n=1}^{N_{\max }} \log _{2}\left(1+\frac{P_{n}}{\Gamma \cdot N_{0} \cdot \Delta f} \cdot e^{-\left(\frac{f_{n}}{f_{0}}\right)^{2}}\right)\right\} \\
& -\lambda \cdot\left(P_{t}-\sum_{n=1}^{N_{\max }} P_{n}\right)
\end{aligned}
$$

Considering the partial derivative of the power in $n^{\text {th }}$ subcarrier:

$$
\frac{d F}{d P_{n}}=\frac{\frac{e^{-\left(\frac{f_{n}}{f_{0}}\right)^{2}}}{\Gamma \cdot N_{0} \cdot \Delta f}}{\left(1+\frac{P_{n}}{\Gamma \cdot N_{0} \cdot \Delta f} \cdot e^{-\left(\frac{f_{n}}{f_{0}}\right)^{2}}\right) \cdot \ln 2}+\lambda=0,
$$

then, we can get that

$$
P_{n}=-\frac{1}{\lambda \cdot \ln 2}-\Gamma \cdot N_{0} \cdot \Delta f \cdot e^{\left(\frac{f_{n}}{f_{0}}\right)^{2}},
$$

Considering the partial derivative of $\lambda$ :

$$
\frac{d F}{d \lambda}=-P_{t}+\sum_{n=1}^{N_{\max }} P_{n}=0
$$

Bringing the result of $P_{n}$ in Eq. (30) then:

$$
P_{t}=\sum_{n=1}^{N_{\max }}\left(-\frac{1}{\lambda \cdot \ln 2}-\Gamma \cdot N_{0} \cdot \Delta f \cdot e^{\left(\frac{f_{n}}{f_{0}}\right)^{2}}\right) .
$$

Then

$$
P_{t}=-\frac{N_{\max }}{\lambda \cdot \ln 2}-\sum_{n=1}^{N_{\max }} \Gamma \cdot N_{0} \cdot \Delta f \cdot e^{\left(\frac{f_{n}}{f_{0}}\right)^{2}} .
$$

Introducing the "imaginary error function" $\operatorname{erfi}(x)$ and Dawson function $D_{+}(x)$. The Dawson function is closely related to the error function $\operatorname{erf}(x)$ :

$$
D_{+}(x)=e^{-x^{2}} \cdot \int_{0}^{x} e^{t^{2}} d t=\frac{\sqrt{\pi}}{2} \cdot e^{-x^{2}} \cdot \operatorname{erfi}(x) .
$$

We introduce the Rectangle rule in Newton-Cotes formulae [29] in order to approximate the continuous definite integral with discrete formula, then Eq. (33) can be represented as:

$$
\begin{aligned}
P_{t} \approx & -\frac{N_{\max }}{\lambda \cdot \ln 2}-\Gamma \cdot N_{0} \cdot \Delta f \cdot \frac{\sqrt{\pi}}{2} \cdot N_{\max } \cdot \frac{f_{0}}{f_{\max }} \\
& \cdot\left[\operatorname{erfi}\left(\frac{f_{\max }}{f_{0}}\right)\right] .
\end{aligned}
$$

Then we can get the Lagrange multiplier variable $\lambda$ :

$$
\lambda=-\frac{N_{\max }}{\left(P_{t}+\Delta\right) \cdot \ln 2},
$$

where $\Delta=\Gamma \cdot N_{0} \cdot \Delta f \cdot \frac{\sqrt{\pi}}{2} \cdot N_{\max } \cdot \frac{f_{0}}{f_{\max }} \cdot\left[\operatorname{erfi}\left(\frac{f_{\max }}{f_{0}}\right)\right]$. 
Finally, bringing the result of $\lambda$ in Eq. (30), we can get the optimal power allocated to each subcarrier:

$$
P_{n}=\frac{P_{t}+\Delta}{N_{\max }}-\Gamma \cdot N_{0} \cdot \Delta f \cdot e^{\left(\frac{f_{n}}{f_{0}}\right)^{2}} .
$$

The optimal transmission rate $R$ is

$$
\begin{aligned}
R= & N_{\max } \cdot \log _{2}\left(\frac{\left(P_{t}+\Delta\right)}{\Gamma \cdot N_{0} \cdot \Delta f \cdot N_{\max }}\right) \\
& -\frac{1}{\ln 2} \cdot \sum_{n=1}^{N_{\max }}\left(\frac{f_{n}}{f_{0}}\right)^{2} .
\end{aligned}
$$

Expending the summary in Eq. (38), the optimal transmission rate $R$ can be achieved:

$$
\begin{aligned}
R= & N_{\max } \cdot \log _{2}\left(\frac{\left(P_{t}+\Delta\right)}{\Gamma \cdot N_{0} \cdot \Delta f \cdot N_{\max }}\right)-\frac{\Delta f^{2}}{\ln 2 \cdot N^{2} \cdot f_{0}{ }^{2}} . \\
& \frac{1}{6} \cdot N_{\max } \cdot\left(N_{\max }+1\right) \cdot\left(2 N_{\max }+1\right)
\end{aligned}
$$

The number of maximal used bandwidth $N_{\max }$ is:

$$
\begin{gathered}
N_{\max }=\max (n) \text { s.t. } \\
\frac{P_{t}+\Delta}{n}-\Gamma \cdot N_{0} \cdot \Delta f \cdot e^{\left(\frac{f_{n}}{f_{0}}\right)^{2}} \geq 0
\end{gathered}
$$

completing the proof.

\section{APPENDIX B}

Maximization of the Bit-Rate with LA Expression

The problem of the optimization is mentioned as follows:

$\operatorname{Max} .\left\{\sum_{n=1}^{N_{\max }}\left(10 \cdot \log _{10}\left(\frac{P_{n}}{N_{0} \cdot \Delta f} \cdot e^{-\left(\frac{f_{n}}{f_{0}}\right)^{2}}\right)-A_{2}\right) / A_{1}\right\}$,

under the constrained condition:

$$
\sum_{n=1}^{N_{\max }} P_{n}=P_{t}
$$

Introducing variable $\lambda$ for Lagrange multiplier,

$$
\begin{aligned}
& F(\underbrace{P_{n}}_{n=1,2 \ldots, N_{\max }}, \lambda) \\
& =\left\{\sum_{n=1}^{N_{\max }}\left(10 \cdot \log _{10}\left(\frac{P_{n}}{N_{0} \cdot \Delta f} \cdot e^{-\left(\frac{f_{n}}{f_{0}}\right)^{2}}\right)-A_{2}\right) / A_{1}\right\} \\
& \\
& -\lambda \cdot\left(P_{t}-\sum_{n=1}^{N_{\max }} P_{n}\right),
\end{aligned}
$$

Considering partial derivative of the power in $n^{\text {th }}$ subcarrier:

$$
\frac{d F}{d P_{n}}=\frac{10}{A_{1} \cdot \ln 10 \cdot P_{n}}+\lambda=0
$$

then

$$
P_{n}=-\frac{10}{\lambda \cdot A_{1} \cdot \ln 10}
$$

Considering the partial derivative of $\lambda$ :

$$
\frac{d F}{d \lambda}=-P_{t}+\sum_{n=1}^{N_{\max }} P_{n}=0
$$

Bringing the result of $P_{n}$ in Eq. (45), then:

$$
P_{t}=\sum_{n=1}^{N_{\max }}\left(-\frac{10}{\lambda \cdot A_{1} \cdot \ln 10}\right)=-\frac{N_{\max } \cdot 10}{\lambda \cdot A_{1} \cdot \ln 10}
$$

Then we can get the Lagrange multiplier variable $\lambda$ :

$$
\lambda=-\frac{N_{\max } \cdot 10}{P_{t} \cdot A_{1} \cdot \ln 10} .
$$

Finally, bringing the result of $\lambda$ in Eq. (45), we can get the optimal power allocated to each subcarrier:

$$
P_{n}=\frac{P_{t}}{N_{\max }} .
$$

The optimal transmission rate $R$ is:

$$
\begin{aligned}
R= & N_{\max } \cdot\left[\frac{10}{A_{1}} \cdot \log _{10}\left(\frac{P_{t}}{N_{\max } \cdot N_{0} \cdot \Delta f}\right)-\frac{A_{2}}{A_{1}}\right] \\
& -\frac{10}{A_{1} \cdot \ln 10} \sum_{n=0}^{N_{\max }}\left(\frac{f_{n}}{f_{0}}\right)^{2} .
\end{aligned}
$$

Expending the summary in Eq. (50), the optimal transmission rate $R$ can be achieved:

$$
\begin{aligned}
R= & N_{\max } \cdot\left[\frac{10}{A_{1}} \cdot \log _{10}\left(\frac{P_{t}}{N_{\max } \cdot N_{0} \cdot \Delta f}\right)-\frac{A_{2}}{A_{1}}\right] \\
& -\frac{10 \cdot \Delta f^{2}}{A_{1} \cdot \ln 10 \cdot N^{2} \cdot f_{0}^{2}} \cdot \frac{1}{6} \cdot N_{\max } \cdot\left(N_{\max }+1\right) \\
& \cdot\left(2 N_{\max }+1\right) .
\end{aligned}
$$

The number of maximal used bandwidth $N_{\max }$ is

$$
\begin{gathered}
N_{\max }=\max (n) \quad s . t . \\
10 \cdot \log _{10}\left(\frac{P_{n}}{N_{0} \cdot \Delta f} \cdot e^{-\left(\frac{f_{n}}{f_{0}}\right)^{2}}\right)-A_{2} \geq 0,
\end{gathered}
$$

completing the proof.

\section{REFERENCES}

[1] C.M. Okonkwo, E. Tangdiongga, H. Yang, D. Visani, S. Loquai, R. Kruglov, B. Charbonnier, M. Ouzzif, I. Greiss, O. Ziemann, R. Gaudino, and A.M.J Koonen, "Recent Results from the EU POF-PLUS Project: Multi-Gigabit Transmission over $1 \mathrm{~mm}$ Core Diameter Plastic Optical Fibers", Journal of Lightwave Technology, vol. 29, no. 2, pp. 186-193, Jan. 152011.

[2] O. Ziemann, J. Krauser, P.E. Zamzow, and W. Daum, "POF Handbook: Optical Short Range Transmission Systems", 2nd Edition, Springer, 2008. 
[3] S.C.J. Lee, F. Breyer, S. Randel, R. Gaudino, G. Bosco, A. Bluschke, M. Matthews, P. Rietzsch, R. Steglich, H.P.A. van den Boom, and A.M.J. Koonen, "Discrete Multitone Modulation for MaximizingTransmission Rate in Step-IndexPlastic Optical Fibers", Journal of Lightwave Technology, vol. 27, no. 11, pp. 1503-1513, Jun. 12009.

[4] S. Randel, F. Breyer, S.C.J. Lee, and J.W. Walewski, "Advanced Modulation Schemes for Short-Range Optical Communications", IEEE Sel. Topics in Quantum Electronics, vol. 16, no. 5, pp. 1280-1289, Sep-Oct 2010.

[5] D. Visani, C. Okonkwo, S. Loquai,H. Yang, Y. Shi, H.P.A. van den Boom, T. Ditewig, G. Tartarini, B. Schmauss, S.C.J Lee, A.M.J. Koonen, and E. Tangdiongga, "Beyond $1 \mathrm{Gbit} / \mathrm{s}$ Transmission Over $1 \mathrm{~mm}$ Diameter Plastic Optical Fiber Employing DMT for In-Home Communication Systems", Journal of Lightwave Technology, vol. 29, no. 4, pp. 622-628, Feb. 152011.

[6] Asymmetric Digital Subscriber Line (ADSL) Transceivers, ITU Std. G.992.1, July 1999.

[7] www.ict-pof-plus.eu (POF-PLUS project web-site).

[8] S.C.J. Lee, F. Breyer, S. Randel, O. Ziemann, H.P.A. van den Boom, and A.M.J. Koonen, "Low-Cost and Robust 1-Gbit/s Plastic Optical Fiber Link Based on Light-Emitting Diode Technology", OFC/NFOEC 2008.

[9] B. Charbonnier, P. Urvoas, M. Ouzzif, J. Le Masson, J. D. Lambkin, M. O'Gorman and R. Gaudino, "EU project POF-PLUS: Gigabit Transmission over $50 \mathrm{~m}$ of Step-index Plastic Optical Fibre for Home Networking", OSA/OFC/NFOEC 2009.

[10] B. Cardiff, M.F. Flanagan, F. Smyth, L.P. Barry and A.D. Fagan, "On Bit and Power Loading for OFDM Over SI-POF", Journal of Lightwave Technology, vol. 29, no. 10, pp. 1547-1554, May 152011.

[11] R. Gaudino, G. Bosco, A. Bluschke, O. Hofmann, N. Kiss,M.Matthews, P. Rietzsch, S. Randel, J. Lee, and F. Breyer, "On the ultimate capacity of SI-POF links and the use of OFDM: Recent results from the POF-ALL project," Fiber OpticsWeekly Update, pp. 283-288, 2007.

[12] J. Campello, "Practical Bit Loading for DMT", ICC 1999.

[13] H.E. Levin, "A complete and optimal data allocation method for practical discrete multitone systems", GLOBECOM 2001.

[14] P.S. Chow, J.M. Cioffi, and J.A.C. Bingham, "A Practical Discrete Multitone Transceiver Loading Algorithm for Data Transmission over Spectrally Shaped Channels", IEEE Transactions on Communications, vol. 43, no. 234, pp. 773-775, Feb/Mar/Apr 1995.

[15] H. Yang, S. C. J. Lee, E. Tangdiongga, C. Okonkwo, H.P.A. van den Boom, F. Breyer, S. Randel, and A. M. J. Koonen, "47.4 Gb/s Transmission Over 100 m Graded-IndexPlastic Optical Fiber Based on Rate-AdaptiveDiscrete Multitone Modulation", Journal of Lightwave Technology, vol. 28, no. 4, pp. 352-359, Feb. 152010.

[16] A. Czylwik, "Adaptive OFDM for wideband radio channels", GLOBECOM 1996.

[17] P.K. Vitthaladevuni and M.S. Alouini, "Exact BER Computation for the Cross 32-QAM Constellation", International Symposium on Control, Communications and Signal Processing, 2004.

[18] C. E. Shannon, "A mathematical theory of communication," Bell Syst. Tech. J., vol. 27, pp. 379-423, 623-656, Jul., Oct. 1948.

[19] A.G. Armada, "SNR Gap Approximation for M-PSK-Based Bit Loading”, IEEE Transactions on Wireless Communications, vol. 5, no.1, Jan. 2006.

[20] S. Loquai, R. Kruglov, C.A. Bunge, O. Ziemann, B. Schmauss, and J. Vinogradov, "10.7-Gb/s Discrete Multitone Transmission Over 25-m Bend-Insensitive Multicore Polymer Optical Fiber", IEEE Photonics Technology Letters, vol. 22, no. 21, pp. 1604-1606, Nov. 12010.

[21] G. Cossu, A. M. Khalid, P. Choudhury, R. Corsini and E. Ciaramella, "2.1 Gbit/s Visible Optical Wireless Transmission”, ECOC 2012.

[22] K. Cho and D. Yoon, "On the General BER Expression of One- and Two-Dimensional Amplitude Modulations", IEEE Transactions on Communications, vol. 50, no. 7, pp. 1074-1080, Jul. 2002.
[23] A. Bjorck, "Numerical Methods for Least Squares Problems", Society for Industrial and Applied Mathematics, 1996.

[24] L. Peng, S. Haese, M. Helard, "Optimized Discrete Multi-tone Communication over Polymer Optical Fiber", to appear in Journal of Optical Communications and Networking, vol. 5 no. 11, 2013.

[25] I.B. Vapnyarskii, "Lagrange multipliers", Encyclopedia of Mathematics, Springer, 2012.

[26] I. Neokosmidis, T. Kamalakis, J.W. Walewski, B. Inan, and T. Sphicopoulos, "Impact of Nonlinear LED Transfer Function on Discrete Multitone Modulation: Analytical Approach", Journal of Lightwave Technology, vol. 27, no. 22, pp. 4970-4978, Nov. 152009.

[27] L. Peng, S. Haese, and M. Helard, "Frequency Domain LED Compensation for Nonlinearity Mitigation in DMT Systems", IEEE Photonics Technology Letters, vol. 25, no. 20, pp. 2022-2025, Oct. 2013.

[28] P. M. Mulligan, "POF Connectors: A review of what's available", ECOC 2007.

[29] P.J. Davis, P. Rabinowitz, "Methods of numerical integration", Acad. Press, 1984.

Linning Peng received his B.S. and M.S. degrees in 2006 and 2010 respectively, from Institute of Information Science and Engineering, Southeast University, China. He is now a $\mathrm{PhD}$ student in IETR (Electronics and Telecommunications Institute of Rennes) laboratory at INSA (National Institute of Applied Sciences) of Rennes, France, from 2010. His primary research interests focus on optical communications and indoor optical networking.

Maryline Hélard received the M.Sc and $\mathrm{PhD}$ degrees from INSA (National Institute of Applied Sciences) of Rennes and the Habilitation degree from Rennes 1 University in 1981, 1884 and 2004 respectively. In 1985, she joined France Telecom Research Laboratory as a research engineer and since 1991 she carried out physical layer studies in the field of digital television and wireless communications. In 2007, she joined INSA as a professor and she is now the co-director of the Communication Department at IETR (Electronics and Telecommunications Institute of Rennes). She is co-author of 22 patents and several papers (Journal and conferences). Her current research interests are in the areas of digital communications such as equalization, synchronization, iterative processing, OFDM, MC-CDMA, channel estimation, and MIMO techniques applied to wireless communications and more recently to wire communications (ADSL, optical)..

Sylvain Haese received the engineer and Ph.D degrees in electrical engineering from INSA (National Institute of Applied Sciences) Rennes, France, in 1983 and 1997 respectively. From 1984 to 1993 he was an analog IC designer for automotive and RF circuits. In 1993, he joined INSA/IETR (Electronics and Telecommunications Institute of Rennes) laboratory where he conducted research for automotive powerline applications and for RF wideband channel sounder circuitry. He is currently involved in hardware analog implementation for RF and optical circuits with the Communication Department at IETR Rennes. 